\title{
Collective Intelligence for Enhanced Quality Management of IT Services
}

\author{
Maja Vukovic and Arjun Natarajan \\ IBM T.J. Watson Research, 19 Skyline Drive, Hawthorne, NY 10532, USA \\ \{maja, arjunn\} @us.ibm.com
}

\begin{abstract}
Customer satisfaction and delivery excellence measure the overall quality of IT services. Services quality management relies on the insights obtained by extracting large volumes of tacit knowledge about processes, products and people. This knowledge is not automatically discoverable, as it is unstructured and widely distributed among the experts, making it challenging to drive quality across all these dimensions. To address these knowledge gaps needed for next level of quality management of IT services we apply collective intelligence methodology, by engaging a set of experts to discover knowledge through collaboration. We further augment enterprise data sources with uncovered human knowledge. We demonstrate the effectiveness of our approach addressing challenges in scalable knowledge discovery both as part of large-scale business transformational and on-going operational activities.
\end{abstract}

Keywords: Services Quality, IT Service Delivery, Collective Intelligence.

\section{Introduction}

IT outsourcing enables companies to contract out IT services, such as infrastructure management, to external providers. It attracted enterprises given a cost reduction and steep improvement in quality through aggressive service level agreements (SLAs) $[1,2]$. As IT outsourcing matured, quality of service became a differentiating factor for providers. To meet the quality expectations and reduce operating costs the providers need to continuously improve services quality both at front-end (e.g., client experience and satisfaction), and back-end (e.g. production and delivery).

IT outsourcing is a complex and extremely dynamic ecosystem. As shown on Fig. 1, typical IT service delivery environment consists of $1000 \mathrm{~s}$ of products (e.g. middleware), each with 100s of instances and configurations [3]. Moreover, there are 100000 s people in delivery centers globally supporting 1000s of processes, such as patch management and backup failure management. At that scale and complexity driving quality across all dimensions and in global context, becomes challenging.

Success of quality management in manufacturing relies on understanding of the back-end processes, and modularizing their operations. In contrast, IT services have experiential nature and require user practice insights. For example, while a database configuration can be automatically extracted, it is the expert who knows how it is used and its importance. Similarly, although processes document the operation flows, it is the delivery experts who understand where the bottlenecks are. 


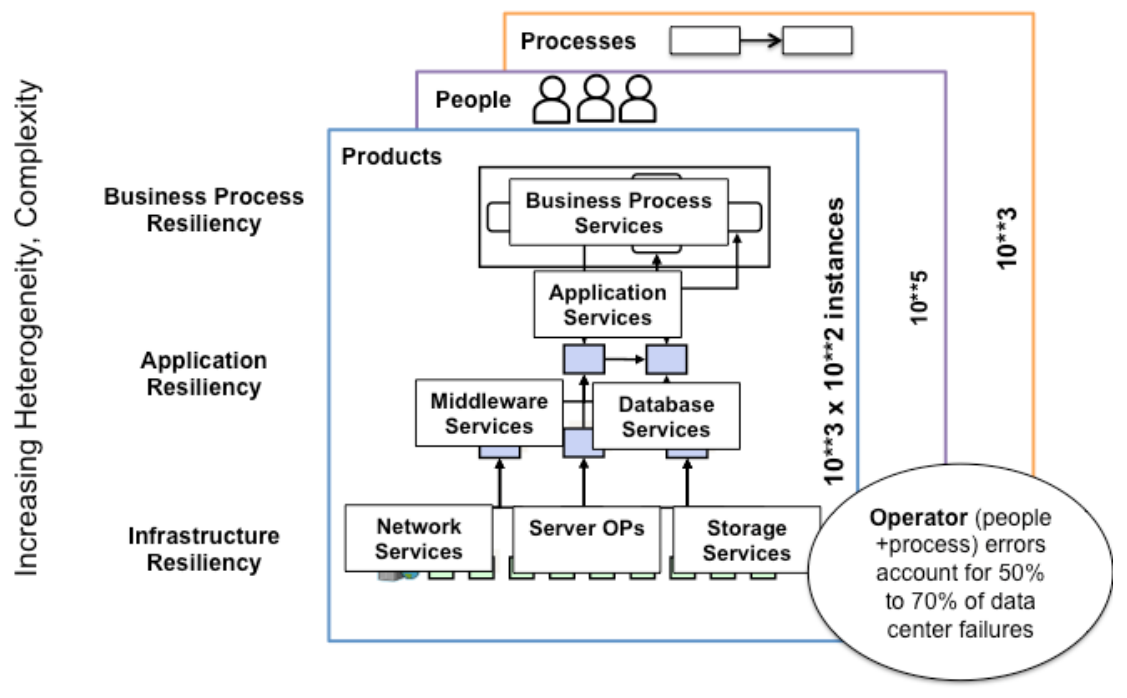

Fig. 1. IT Services Delivery - Process, Product and People [3]

Collective intelligence refers to a group of experts that through collaboration make decisions and solve problems as a (distributed) team. Xerox's Eureka system is one of the early examples of applications of collective intelligence for IT support services [4], which enabled experts to share best practices. To increase service quality in IT outsourcing, by accelerating knowledge discovery, we propose end-to-end integration of collective intelligence with existing process operations. As opposed to an "opencall" approach, we select participants based on their expertise.

In this paper we present how we integrated collective intelligence of experts using BizRay crowdsourcing service [5] with enterprise data systems, to derive quality insights about products, processes and people in IT services. Next section introduces background on IT service quality. Section 3 describes the integration of human and digital knowledge. Section 4 presents applications of our approach to harvest the knowledge about products, people and processes in IT services. Section 5 discusses results from discovering knowledge about defect prevention tools from $2300+$ quality analysts, automation opportunities from 90 system administrators and compliance insights from 128 delivery staff. Section 6 puts our work in the context of state of the art in service quality and collective intelligence. Section 7 sets our future directions and concludes.

\section{Complexity of IT Service and Quality Management Challenges}

\subsection{Background}

IT outsourcing services consist of a stack of functions such as, infrastructure management and help-desk operations. An IT outsourcing provider runs operation and maintenance of client's IT environment, with the goal of improving efficiency 
through quality of production and delivery. In large deals the IT outsourcing provider typically takes over the entire set of client's IT functions, in contrast to smaller deals where it assumes operations of only a single function such as help-desk operations. Contract between provider and consumer outlines the outsourcing model and (quality) metrics to be continuously verified, known as Service Level Agreement (SLA).

IT services evolved from isolated environments in 1980's where there was no formal separation between the users of IT and the providers. In the 1990's services were becoming more componentized and shared, and operations formalized given the emergence of standards. In the 2000's global delivery model was widely adopted, and new challenges were introduced given the huge separation between provider and consumer, time zone differences, language barriers, etc. Complexity of service systems is evolving given the diversity of providers and consumer. This impacts how we get services to work together and how we measure them. In the 2010's we are witnessing a dramatic growth in number of service providers and granularity of services offered with inconsistent service levels, given a low barrier to entry. No single entity owns the entire service system. Lack of standard metrics and centralized ground truth source results in IT services based on inconsistent and conflicting data.

\subsection{Quality of IT Services}

With the increasing complexity of IT Service ecosystem and maturity of IT, quality of service became a differentiating factor for providers. Client satisfaction is an increasingly dominating choice of service provider. For example, $59 \%$ of respondents in the Accenture's study report switching from at least one provider within a year due to poor service [6].

There are two distinct aspects to measuring services quality: a service provider perspective and a service consumer perspective. Quality metrics in product industries tend to be producer centric, whereas in services the consumer perspective is as important if not dominant. Consumer perception includes the client value and SLAs. The provider quality is measured using SLA attainment, number of defects and financial aspects of operations. On the service provider side, key measure employs General Sigma approach to drive service production quality. "Defect" can be defined as an instance or event that is not a satisfactory outcome. On the consumer side, traditional Sigma approach is less understood. The consumer measure represents the difference between consumers' perception and expectation. Notion of "defect" is harder to define and carries a significant subjective component.

Consumer and provider perspective around the same service are often quite different. For example, an airline industry study revealed the despite airline baggage handling being at nearly $4 \operatorname{sigma}^{1}[7]$, it was still the $2^{\text {nd }}$ highest passenger complaint [8].

While quality engineering methodology can be adopted from established fields such as manufacturing, IT Services Quality has fundamental new dimensions related to the experiential nature and co-production of services with the client. As such it requires focus on integrated product-based process-driven, and people-centric operations.

\footnotetext{
1 "Sigma" is around a specific metric. A service system has a number of metrics reflecting processes and tasks across the lifecycle. These metrics can range between 2 and 6 sigma as business criticality of individual metrics vary.
} 
From product perspective it is important to understand how products (and tools) are used as part of IT services delivery processes. For example, in the defect prevention, addressed in Section 4.1, quality analysts rely on tools to classify and expedite the problem resolution. The quality depends on the usage and usability of available tools. Opportunities for process quality improvements are associated with automation. In Section 4.2, we investigate a set of work activities in delivery centers to identify their current levels of performance and discover further automation opportunities. Quality enhancements arising from people centric insights are two fold. First is the improved compliance posture of business described in Section 4.3. Second benefit is the ability to allocate experts more efficiently based on their expertise.

\subsection{Front and Backend Elements of IT Service}

Service systems can be conceptualized as a stage [9], with front-end (client-facing) and back-end (operations) functions, shown on Figure 2. A lower level, back-end stage, that focuses on consistent delivery through standardization, automaton and a learning system for continuous improvement. The middle level glues the loop between the front-stage and the back-stage. The top level, the front end, focuses on the client by developing a branded services experience and client value. Metrics at each level form a "network of metrics" determining overall quality of the service. For IT Services Delivery to take advantage of advances in manufacturing industry, a factory model has been applied to the back-end operations. This results in standardized, automated processes with adaptive dispatching (tasks sent to experts based on domain and complexity). Experts are grouped into "pools" sharing a specific competency. The delivery knowledge is captured into best practices, and real-time metrics provide sensing and response capabilities to drive continuous improvement. Finally, a learning system prevents repeat incidents leading to self-healing.
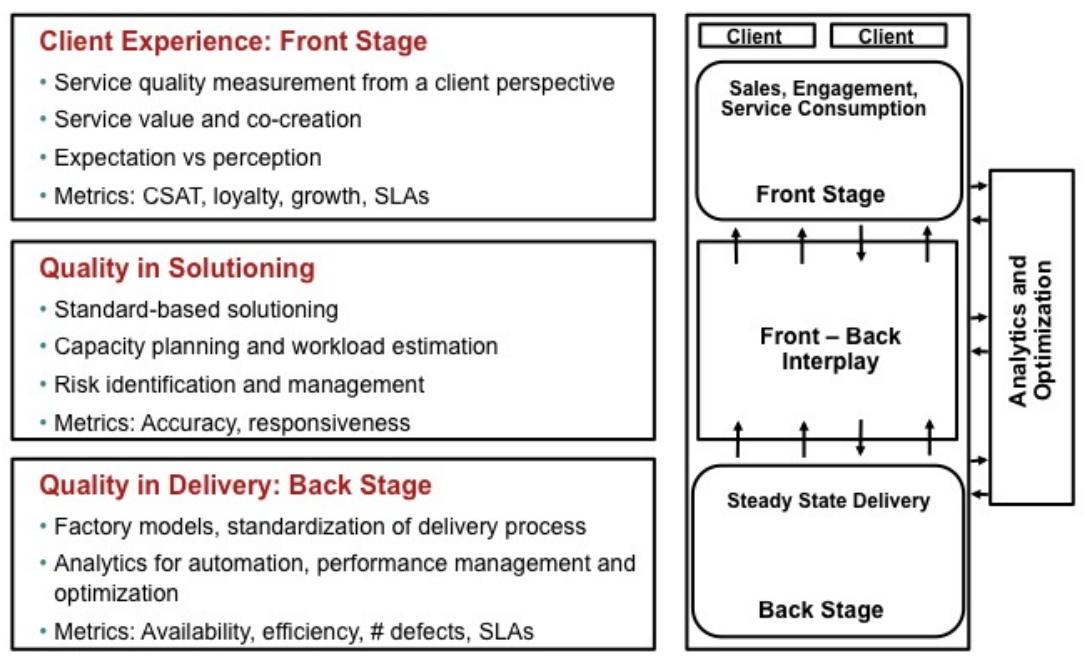

Fig. 2. Quality Characteristics of IT Services at Front and Back-end [3] 


\section{Approach: Integrating Digital and Collective Intelligence}

Knowledge discovery is a distributed process often relying on multiple human or digital sources. Traditional techniques for knowledge discovery in enterprises such as interviews, e-mails, instant messages and Web-forms are no longer sufficient to reach out to today's globally distributed teams in large enterprises. They fall short in systematically tracing the responses, dealing with incomplete responses, and understanding the organizational relationships, with Subject Matter Experts (SMEs) moving out of organization. SMEs may forward knowledge requests to experts who took over their previous roles, yet these methods are time-consuming and intractable.

There are two critical factors that impact success of the knowledge discovery: knowing the potential source, or knowing someone who is aware of other sources. Crowdsourcing gained popularity with the emergence of Web 2.0, enabling enterprises to outsource tasks that are traditionally performed by designated human agents to an undefined large group of humans [10]. Even within the enterprise it can be applied in the form of an open call format allowing for anyone to participate. For instance, when looking for information about Apache Tomcat build procedure it may be useful to setup a forum for people interested. Yet, when seeking accurate information on a business entity, the potential contributors that may answer correctly are much smaller and may not be necessarily available for such inquiries. In this case it is easier to establish chains of inquiries.

To implement principles of collaborative knowledge discovery (Figure 3.) in Services Delivery we employ system BizRay [5]. Knowledge requests are captured in the distributed questionnaire artifact, which consists of one or more sections, each of which consists of one or more questions. BizRay manages its lifecycle, similar to a workflow system and facilitates delegation of requests and their subtasking. More than one expert (user) can complete each questionnaire instance. If the information gathered is incomplete or unidentified, the user can forward the request to another expert, asking for their help. As experts contribute their knowledge, the system keeps track of their identity resulting in the formation of micro communities around the object of that inquiry. The system also embeds capabilities to send out reminders and escalations to users who did not respond to the initial request.

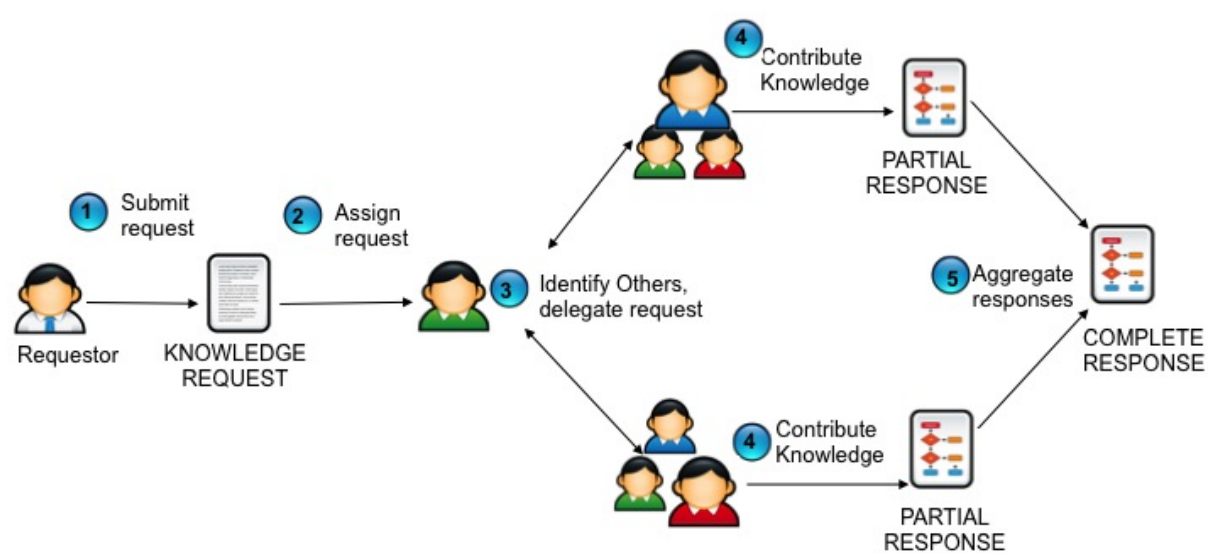

Fig. 3. Collective Intelligence Approach to Knowledge Discovery 


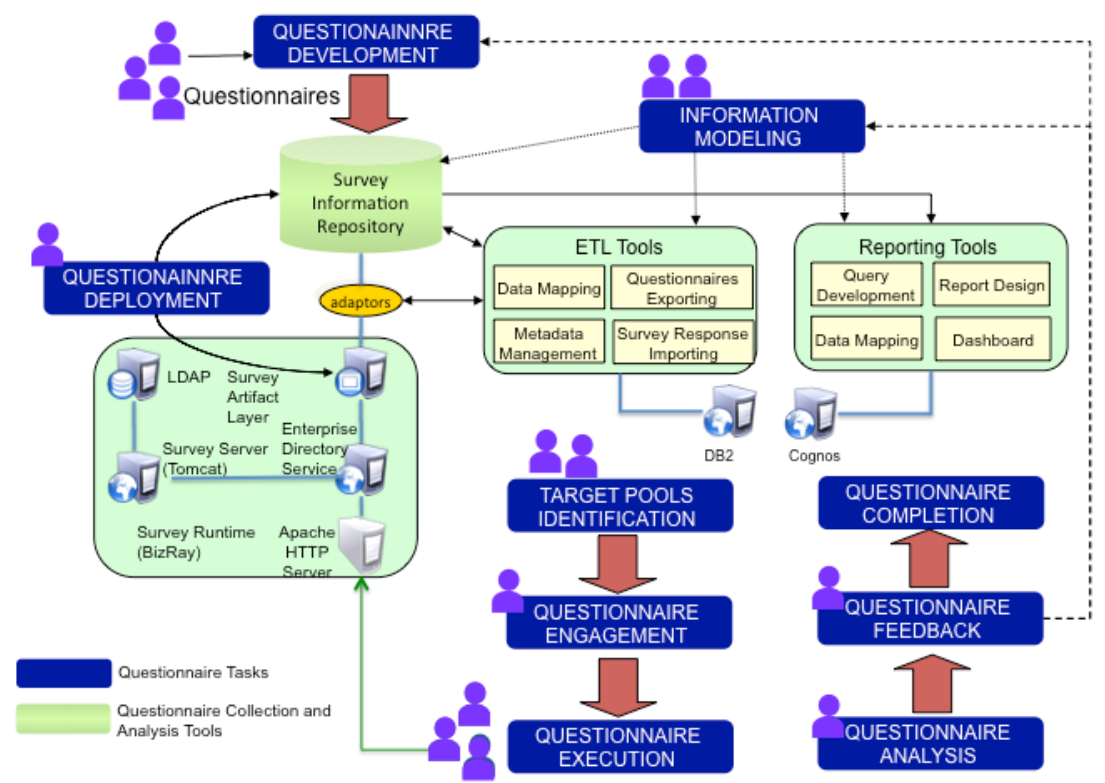

Fig. 4. Integration of Crowdsourcing Results with Enterprise Data Sources

In contrast to our prior work [5] where questionnaires were deployed as isolated instances, we integrated BizRay with existing enterprise data and reporting sources. Figure 4 shows the system architecture and lifecycle, and how we automatically processed questionnaire results, mapping them into the reports that are consumable by business analysts. The centralized information model captures the data for organization, labor, tools, automation potential, etc. Automated ETL tool maps questionnaire to this data model. Automated reporting tool, based on IBM Cognos platform, allows business users to query and view the different elements of questionnaire data. BizRay was used to manage questionnaires, tasks, delegations, status and reports. A feedback loop supports systematic refinement of questionnaires. Open source crowdsourcing solutions can be used to provide core functions, however BizRay has a unique feature that allows users to subtasks their assignments.

\section{$4 \quad$ IT Service Insights for Quality Management}

In this section we describe three applications of collective intelligence to IT Services Delivery operations within a global enterprise, harvesting insights and improving product, processes and people aspects of the back-end processes. 


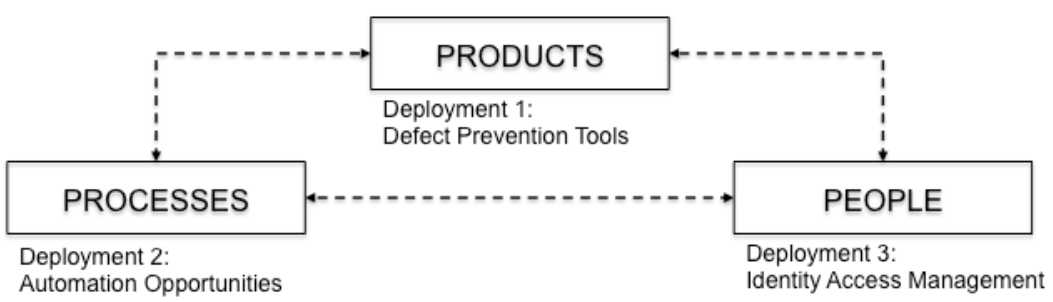

Fig. 5. Dimensions affecting IT Service Quality

\subsection{Product Insights - Defect Prevention Tools}

Defect prevention is the proactive and systematic methodology for improving quality and productivity by preventing the injection of defects into a service. It has three primary objectives: improving quality, improving productivity, and improving solution knowledge management. Improvements to quality in IT services are achieved by targeting defects, understanding the root cause, and eliminating defects by implementing solutions and corrective actions. Productivity improvement is achieved by removing non-value add activities that are involved in defect handling. For example, in IT Outsourcing, significant time is spent resolving recurring incidents and alert disposition. This time can be eliminated and the effort can be spent on activities that add value to the customer. Finally effective knowledge management is achieved by ensuring organizational support to effectively leverage defect knowledge across different outsourcing customers. The objective is that defect prevention leverages globally distributed teams that provide IT services to resolve problems only once and share the solutions globally, thereby improving the overall level of service quality.

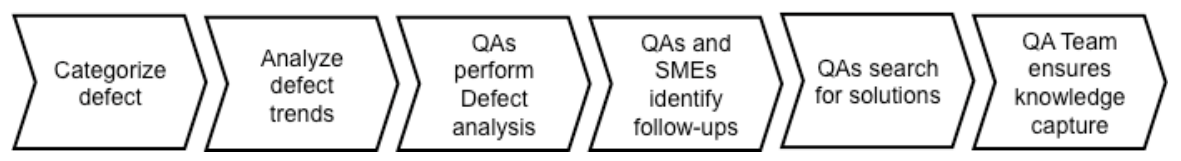

Fig. 6. Defect Prevention Process Flow: Analysis, Solution and Reuse

Figure 6. shows the main stages in defect preventions process, starting with the Quality Analysts (QAs) performing the classification and analysis of incoming issues and trends and identifying follow-up investigations. Subsequently QAs together with Subject Matter Experts (SMEs) and System Administrators (SAs), work on resolving the issues and capturing the best practices.

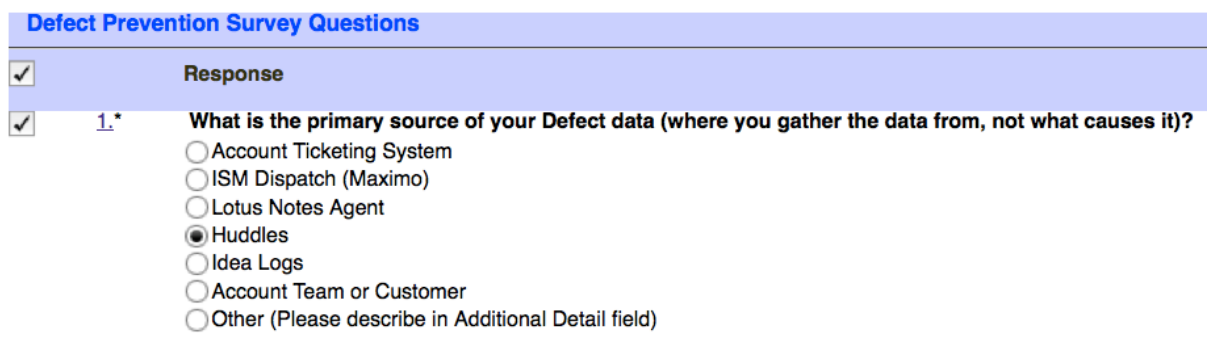

Fig. 7. Defect Prevention Collective Knowledge Request 
We have applied principles of collective intelligence to $2300+$ quality analysts in an effort to understand their practices, usage of available products (tools) for defect prevention and sample opportunity for novel auto-classification capabilities. The estimated time to complete the request, shown in Figure 7., was 5 minutes, and no tangible incentive was offered to participants. The campaign ran for a month, during which we obtained over $50 \%$ responses.

Within first week nearly $30 \%$ of respondents provided their responses. The rate of responses slowed down, and has resurged following a reminder that we have issued after third week. The primary value of the questionnaire is the insights obtained from respondents. It was discovered that over $60 \%$ of the QAs are still manually locating defect codes, opening up an opportunity for introducing advanced methods for autoclassification capabilities with the defect prevention process. Analysis further revealed that $25 \%$ requests were forwarded to another QA, reflecting team changed. Another $20 \%$ of respondents rejected the knowledge request altogether, claiming that they are no longer in this job function. Nevertheless, this data is enabling organization to track the size and distributions of competencies and knowledge capital (i.e. affecting people aspect of service delivery).

\subsection{Process Insights - Automation Opportunities}

Our objective was to discover automation opportunities for service management processes using questionnaires about accounts that are managed by pools (Figure 8) and the tools used. From account perspective our goal was to identify variances among accounts being managed, business constraints and infrastructure prerequisites for automation. From the tool perspective, the goal was to understand the execution time for different work activities, existing automations and the estimated time reduction they introduce. Work-types performed by pool members included resolving hardware issues, patch and backup failure management, etc.

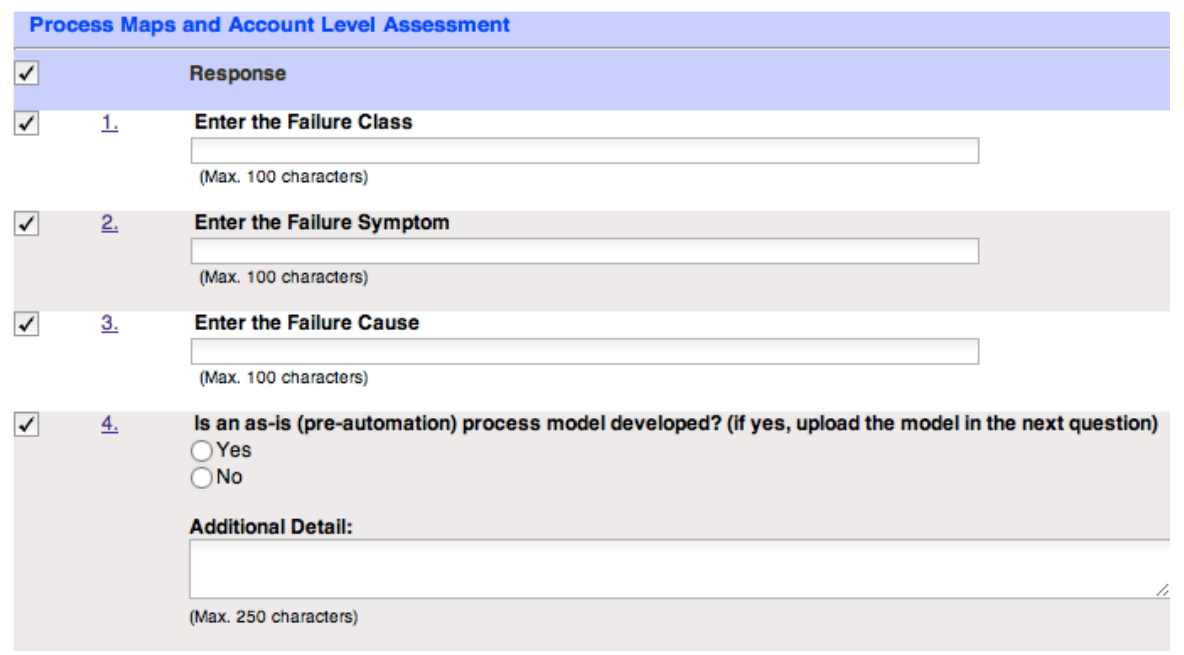

Fig. 8. Account Level Questionnaire for Automation Opportunities 
We were interested in performance of current processes in pools and opportunities for automation globally. As pools often develop their own productivity tools, we wanted to obtain a list of existing assets and identify their synergies. We assigned the questionnaire to SAs for 90 pools, with no explicit incentives.

Analysis of the results provided insights into variance in productivity between different pools that share business objectives, as shown in Figure 9. For work type Hardware Issues two pools report entire different duration estimates, whilst for the first three work types (backup, application and capacity system growth) they are consistent. Based on $30 \%$ of responses (other participants didn't provide this information), the mean time reduction arising from tool automation is $77.35 \mathrm{~min}$ per SME. Section 5 discusses challenges arising from inconsistent responses, missing data, and misinterpreted requests.

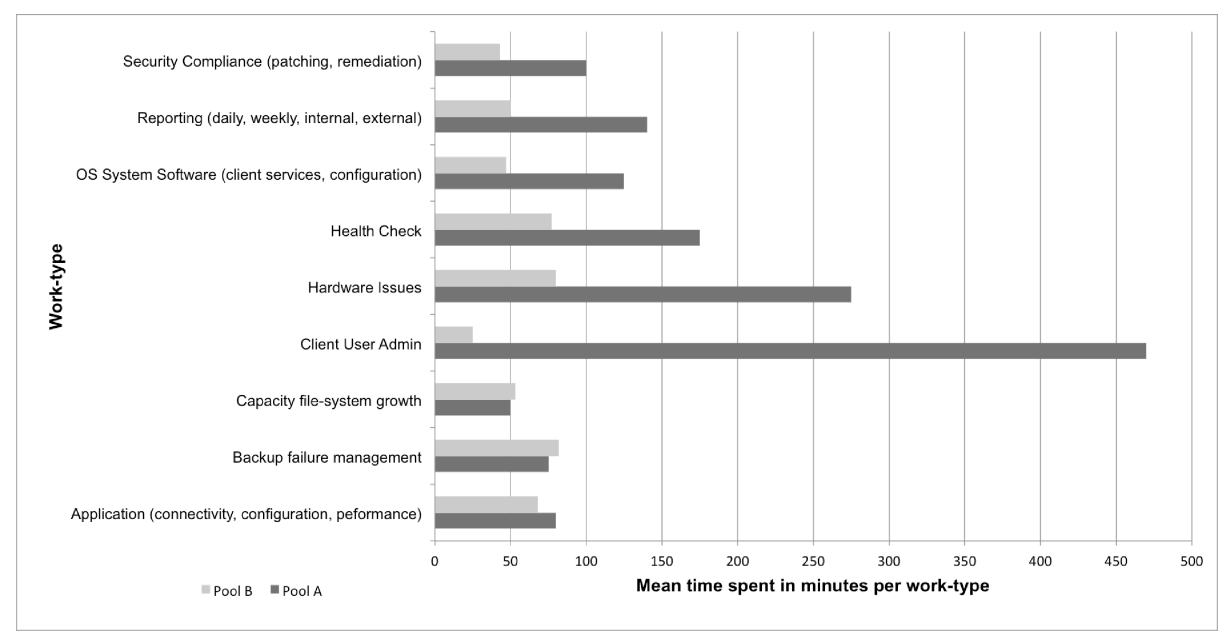

Fig. 9. Operational Insights: Variance of pool performance

\subsection{People Insights - Compliance}

Whilst process automation has a direct impact on the quality, regulated usage and access to customer's data and systems is of utmost concern for any enterprise, let alone IT service provider. Obtaining, managing, executing and disposing of access rights to customer's systems requires structured mechanisms that follow national and industry compliance regulations. Complexity of IT Service environment calls for new approaches to automate system access and provide fine granularity audit records [11].

To continuously improve compliance posture, adhering to a variety of regulations, enterprises require accurate and in-depth insights into employees' existing and required system access rights [12]. To generate comprehensive audit trails enterprises rely on detailed knowledge about how employees access existing systems. For that purpose we have deployed a questionnaire capturing elements of compliance in 128 pools, which were distributed to pool focals. Eighty-nine experts completed the 
request and twenty provided partial response. Due to the complexity questionnaire took on average 13 days to complete (measured from first time access to closure of the questionnaire in the system).

\section{Discussion}

\subsection{Effectiveness of Collective Intelligence}

Figure 10 shows the response time for three different deployments. In the simplest questionnaire (defect prevention) up to $30 \%$ responses were submitted within the first day. We observe bursts of responses as the reminders are sent. BizRay system has minimized human effort in setting up and distributing questionnaire campaigns and has enabled scaling of these activities. This has enabled us to easily reach out to $100 \mathrm{~s}$ or 1000s of SMEs at the same cost.

\subsection{Design Considerations}

Scaling out campaigns impacts the accuracy levels, by introducing inconsistency and incompleteness of the gathered information (e.g. in the Automation Opportunities questionnaire we observed large variance in responses, as a result of ambiguous questions or respondents not providing the response in the correct unit of measure).

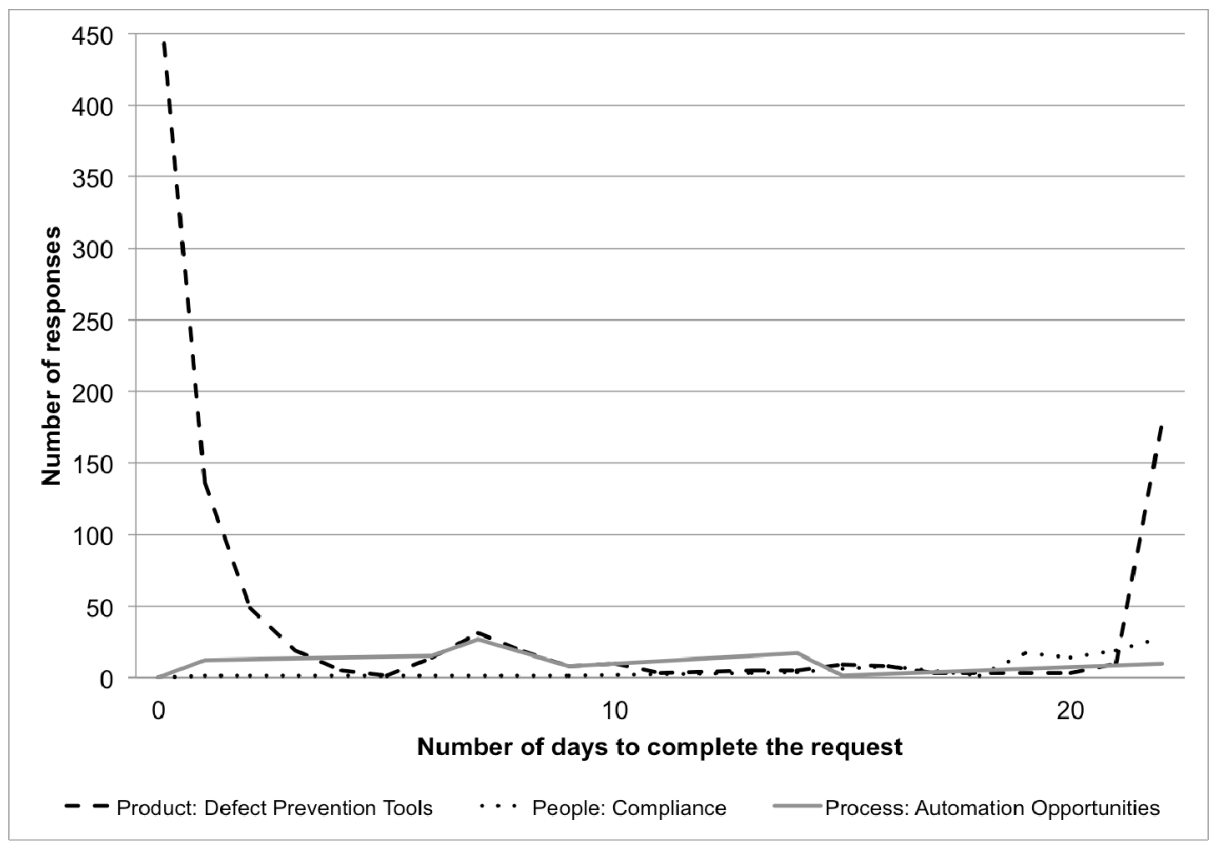

Fig. 10. Distribution of responses 
For example, there was a case where a single SME reported that there is no support for "user admins", the other SME reported that "16hours were spent on supporting user admins", and yet another responded that "15mins were spent on supporting user admins". Anecdotally, the last SME did admit that they were not responsible for that issue resolution, but did spend those 15 minutes helping another colleague. Similarly, respondents may simply be in hurry and hastily respond, entering incorrect data.

However, collective intelligence approach does allow us to follow up with (selected sample) of respondents and verify their responses as part of quality assurance. The ambiguities about the questions can be overcome by facilitating communication among users and questionnaire designer, which indeed the BizRay tool supports.

We have also uncovered conflicts between responses of SMEs of the same pool, for example some were reporting that there is no for Reporting and Health Check, while another stated the names of the tools used for those activities. Normally, only one of them should be right, but here both can be right if they are SME for different accounts within the same pool. As a lesson learnt, we will capture account affiliation of the SME, and further reduce the scope of the question. In addition we discovered that respondents were collaborating on their (individual) questionnaires, and provided similar responses. This may be viewed as a notion of community driven validation.

Table 1. Use of collaborative features

\begin{tabular}{|l|r|r|r|}
\hline \multicolumn{1}{|c|}{ Questionnaire } & $\begin{array}{c}\text { \# Sent } \\
\text { Requests }\end{array}$ & $\begin{array}{c}\text { \# of } \\
\text { Reassignments } \\
\text { (full and partial) }\end{array}$ & $\begin{array}{c}\text { \# of Full } \\
\text { Reassignments }\end{array}$ \\
\hline Product: Defect Prevention & 2334 & 527 & 526 \\
\hline People: Compliance & 128 & 103 & 98 \\
\hline Process: Automation Opportunities & 90 & 6 & 2 \\
\hline
\end{tabular}

\subsection{Implications on Services Quality}

Distributed questionnaires provided dual value: auxiliary and direct [13]. Direct output is driven by the business objectives (e.g. discover automation assets and their effectiveness). Auxiliary output is the derivative, such as discovering new Quality Analysts. Both are critical to improvement of processes (e.g. understanding current operations, and distributions of knowledge capital internally). Table 1 summarizes the usage of collaborative features In the defect prevention scenario the high number of delegations corresponds to the migration of SMEs within the enterprise departments and change in role responsibilities. The communities of experts that were uncovered can be reached out in subsequent campaigns to further expose the products used and procedures followed. This provides an enormous potential in a large global enterprise to further standardize the processes and assets used. 


\subsection{Effectiveness}

In our initial application of BizRay for IT optimization we have demonstrated that introduces up to 30x improvement to data collection process [14]. The results were based on comparison of manually collected data using spreadsheets that were distributed and managed via e-mail. They resulted in manual follow-ups with multiple respondents. In the current work, a comparison is available for automation opportunities. Initially delivery staff was interviewed, for an hour, in person by consultants. Such sessions are often difficult to schedule, as experts need to attend to various high priority tasks. Using BizRay expert can go back multiple times to update their response. BizRay also allowed scaling of the questionnaires without increasing the cost of the effort.

\section{$6 \quad$ Related Work}

We position our work with respect to the state of the art in services quality. We compare our application of collective intelligence to the current state of the art in enterprise crowdsourcing and draw parallels to open source development efforts.

\subsection{Services Quality}

Grover et al. [1] identify high correlation between outsourcing success and system operations functions. Their results indicate that decision to outsource is driven by two types of parameters that impact the overall service quality. First is the quantifiable process improvement potential and related transaction cost. Second are the soft elements of partnership such as trust, cooperation, and communication.

Lee [15] confirms the belief that knowledge sharing is one of the major predictors for outsourcing success and impacts the soft factors of outsourcing partnership. Organizational capability to learn or acquire the needed knowledge from other organizations is a key source of successful knowledge sharing, and partnership quality, impacting the outsourcing success. Zinelid verifies that the strong competitive position is achieved through customer relationship management (CRM) and product/service quality, and proposes a new technical-functional 5 qualities model to measure the quality and loyalty [16]. This breakdown is much aligned with our approach of applying collective intelligence both in the front-end and back-end of services business. Indeed, social media techniques are becoming a prevalent method in reaching out and maintaining the relationship with the customers [17].

\subsection{Collective Intelligence}

Emergence of Web 2.0 and advances in social networking technologies have empowered the potential of collective intelligence to improve quality of business processes and customer relations $[18,19]$. Before Web 2.0 companies were engaging employees through competitions (e.g. employee of the month) to increase 
productivity. Similarly they employed surveys to obtain insights about the potential improvements in the workplace. Using the traditional media enterprises were reaching out to customers to sample ideas for a new product or service or quality improvements. Vukovic and Naik [20] engage enterprise online communities to capture the information required for IT optimization, such as migration to Cloud. On the customer facing side, nowadays we are witnessing a plethora of on-line communities, forums and social media applications to engage customers in product and service design decisions [21,22]. Beyond providing feedback on the service operations, end-users and customers are evolving into a virtual extension of the enterprise, by increasingly being embedded in the customer support processes [23,24].

\subsection{Open Source Development}

In production open source is a methodology that promotes free redistribution and access to an end product's design and implementation details. Prior work has drawn parallels between wisdom of crowd approach and open source [25], which Albers et al. [26] identify as just one of the new Internet-based learning and network collaboration paradigms. A main advantage of open-source model is the concept of concurrent yet different agendas and approaches in production, in contrast to the traditional centralized models of development and operations in enterprises. Moreover end-product, source-material, and documentation resulting from the open source method are available at no cost to the public in the open source approach. With globally distributed, virtual teams becoming the norm in the enterprises [27], the elements of open source method are being applied to internal operations.

\section{$7 \quad$ Future Work and Conclusions}

We have presented a collective intelligence approach to enhancing quality management processes of IT services. The main contribution of this paper is a mechanism to augment the tacit human knowledge with digital knowledge from enterprise data sources to provide enhanced enterprise insights. In contrast prior art considers collective intelligence as a stand-alone effort, disconnected from the encompassing business process.

There is very little research in the quality management of front-end processes in IT Services, opening an opportunity for unique differentiation amongst service providers. Our future work will include engaging of clients and account teams to derive insights about client processes and their quality parameters. This will enable us to close the knowledge gap arising from complexity and dynamics involved in interactions between front and back-end service elements.

Acknowledgements. We thank Jim Laredo and Sriram Rajagopal for contributions to the BizRay system, and Winnie Chang for insights from defect prevention analysis. 


\section{References}

1. Grover, V., Cheon, M.J., Teng, T.C.: The Effect of Service Quality and Partnership on the Outsourcing of Information Systems Functions. Journal of Management Information Systems 12(4), 89-117 (1996)

2. Beulen, E., Van Fenema, P., Currie, W.: From Application Outsourcing to Infrastructure Management: Extending the Offshore Outsourcing Service Portfolio. European Management Journal 23(2), 133-144 (2005)

3. IBM Global Technology Outlook, Services Quality (2009)

4. Bobrow, D.G., Whalen, J.: Community Knowledge Sharing in Practice. The Eureka Story. Journal of the Society of Organizational Learning and MIT Press (2002)

5. Laredo, J., Vukovic, M., Rajagopal, S.: Scalable Knowledge Gathering for NonDiscoverable Information. In: International Conference on Service Oriented Computing (2011)

6. Accenture 2010 Global Consumer Research executive summary (2010),

http: / / www . accenture.com/us-en/Pages /

insight-accenture-customer-satisfaction-survey-2010-

summary.aspx

7. IBM. Beyond the carousel, http: / /www-935.ibm.com/services / us / gbs / bus / pdf / g510-6592-00-baggage.pdf

8. Aviation Consumer Protection Division, http://airconsumer.ost.dot.gov

9. Teboul, J.: Service is Front Stage: Positioning Services for Value Advantage. Palgrave Macmillan (2005)

10. Howe, J.: The Rise of Crowdsourcing. Wired 14(6) (2006)

11. Bhaskaran, K., Hernandez, M., Laredo, J., Luan, L., Ruan, Y., Vukovic, M.: Privileged Identity Management in Enterprise Service-Hosting Environments. In: Proceedings of Network Operations and Management Symposium, NOMS 2012 (2012)

12. Vukovic, M., Giblin, C., Rajagopal, S.: Accelerating the Deployment of Security Service Infrastructure with Collective Intelligence and Analytics. In: IEEE Service Computing Conference, SCC 2012, Honolulu (2012)

13. Vukovic, M., Stewart, O.: Collective Intelligence Applications in IT Services Business. In: IEEE Service Computing Conference, SCC 2012, Honolulu (2012)

14. Vukovic, M., Lopez, M., Laredo, J.: PeopleCloud for the Globally Integrated Enterprise. In: Dan, A., Gittler, F., Toumani, F. (eds.) ICSOC/ServiceWave 2009. LNCS, vol. 6275, pp. 109-114. Springer, Heidelberg (2010)

15. Lee, J.-N.: The Impact of Knowledge Sharing, Organizational Capability and Partnership Quality on IS Outsourcing Success. Information Management (2001)

16. Zineldin, M.: The Royalty of Loyalty: CRM, quality and retention. Journal of Consumer Marketing 23(7), 430-437 (2006)

17. Sarner, A., Thompson, E., Dunne, M., Davies, J.: Top Use Cases and Benefits for Successful Social CRM. Gartner White Paper G00209091 (2010)

18. Surowiecki, J.: The Wisdom of Crowds. Anchor (2005)

19. Brabham, D.C.: Crowdsourced advertising: How we outperform Madison Avenue. Flow: A Critical Forum on Television and Media Culture (2009)

20. Vukovic, M., Naik, V.: Managing Enterprise IT Systems Using Online Communities. In: IEEE Services Computing Conference, SCC (2011)

21. Getsatisfacation.com Online Community Software, http: / / www getsatisfaction. com

22. FixYa Solutions for Everything, http://www. fixya.com 
23. CrowdEngineering, http: //www. crowdengineering.com

24. giffgaff - the mobile network run by you, http://www.giffgaff.com

25. Brabham, D.: Crowdsourcing as a model for problem solving: An introduction and cases. Convergence: The International Journal of Research into New Media Technologies (2008)

26. Albors, J., Ramos, J.C., Hervas, J.L.: New Learning Network Paradigms: Communities of Objectives, Crowdsourcing, Wikis and Open Source. International Journal of Information Management 28(3), 194-202 (2008)

27. Malone, T.: The Future of Work. How the New Order of Business Will Shape Your Organization, Your Management Style and Your Life. Harvard Business School Press (2004) 